\title{
Multiple Relapses of Parathyroid Carcinoma with Severe Systemic Complications - Case Report and Literature Review
}

\author{
Andreea ILIESIU1,2, Ana-Maria CIONGARIU1,2, Bogdan SOCEA ${ }^{2,3}$, Mihail-Constantin CEAUSU 2,4
}

\begin{abstract}
Parathyroid carcinoma is an exceptionally uncommon endocrine neoplasm, accounting for less than $1 \%$ of parathyroid tumours and also a rare cause of primary hyperparathyroidism. Although this malignant lesion is usually slowly progressive, it is frequently associated with local recurrences and also with metastases involving the local lymph nodes or distant sites.

We present a 59-year-old male patient who developed a parathyroid carcinoma metastasis involving the anterior mediastinal lymph nodes and thymus remnants,

3 years after the primary tumour was identified and treated by surgical excision followed by chemo and radiotherapy. The patient presented with severe, symptomatic hyperparathyroidism and a gamma scan revealed increased uptake hyperfixation in the paratracheal lymph nodes. A lymphadenectomy was performed and the gross examination of the specimen showed a pinkish - white, firm, poorly circumscribed mass. The microscopic examination revealed an epithelial proliferation with a predominantly nodular/solid growth pattern, composed of cells exhibiting moderate nuclear pleomorphism, prominent nucleoli and high mitotic activity, involving two lymph nodes and thymus remnants. Upon immunohistochemical analysis, the proliferation showed positive staining for GATA 3, as well as a high Ki 67 index, whereas TTF 1 and thyroglobulin were negative in the tumour cells. Thus, the diagnosis of metastatic parathyroid carcinoma was established.

The aim of this paper is to gain further knowledge about the histopathological and immunohistochemical features, as well as about the clinical behaviour of parathyroid malignant lesions, especially considering their rarity.
\end{abstract}

Keywords: parathyroid carcinoma, hyperparathyroidism, immunohistochemistry.

'Emergency University Hospital, Bucharest, Romania ${ }^{2}$ "Carol Davila" University of Medicine and Pharmacy, Bucharest, Romania

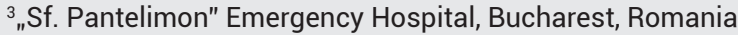
${ }^{4}$ "Alexandru Trestioreanu" National Institute of Oncology,

Bucharest, Romania
Corresponding author.

Ana-Maria CIONGARIU, Emergency University Hospital, „Carol Davila" University of Medicine and Pharmacy, Bucharest, Romania. E-mail: ana.ciongariu@yahoo.com 


\section{INTRODUCTION}

Parathyroid carcinoma is an exceptionally rare neoplasm, accounting for less than $1 \%$ of all cases of primary parathyroid tumours (1). It is considered that this particular malignant epithelial lesion usually has an indolent behaviour with a relatively slowly progressive evolution $^{1,2}$.

The clinical features of parathyroid carcinoma are usually dominated by the signs and symptoms of the elevated secretion of parathormone and its direct effects ${ }^{2}$.

The histopathologic diagnosis of parathyroid carcinoma is challenging mainly because of the difficulty of identifying criteria of malignancy upon conventional microscopic examination and it should only be established based on clear evidence of invasive behaviour involving surrounding structures or if there is evidence of metastases ${ }^{1,3}$. However, metastases can, in exceptionally rare occasions, prove to be primary carcinomas developed on ectopic glandular parathyroid tissue ${ }^{1,3,4}$.

The diagnosis of malignant lesions of the parathyroid gland is particularly challenging and implies histopathological examination and immunohistochemical study, but also a thorough monitoring of the clinical evolution of the disease ${ }^{3,4,5}$.

\section{MATERIALS AND METHODS}

Our patient presented to the Emergency University Hospital in Bucharest, where laboratory tests and Technetium 99 scintigraphy revealed hyperfixation in the paratracheal lymph nodes, for which he underwent a surgical procedure of lymphadenectomy. The specimen was submitted to our Pathology department. The samples were fixed with $10 \%$ neutrally buffered formalin and then processed by conventional histopathological methods, using paraffin embedding, sectioning and Hematoxylin-Eosin (HE) staining. Afterwards, the sections were deparaffinized in toluene and alcohol and then washed in PBS (phosfate saline buffer), incubated with normal serum, then incubated with primary antibody overnight. Later, washing in carbonate buffer and development in 3.3'-diaminobenzidine hydrochloride/hydrogen peroxide and nuclear counterstain with Meyer's Hematoxylin was performed. The immunohistochemical markers that we used were Parathormone, GATA 3, Ki 67, Thyroglobulin and TTF1.

\section{CASE REPORT}

We report the case of a 59-year-old male with no familial history of malignancies. He was firstly admitted in 2018 in a neurology department with complaints of lower back and pelvic pain. An MRI investigation was performed, revealing two heterogenous lesions located in the left iliac wing. Other relevant findings were elevated calcium and PTH levels, renal microlithiasis and multiple foci of osteitis fibrosa cystica. Subsequently, a gamma scan was performed and it showed a hyperfixating nodule in the anterior cervical region, suggesting the presence of a parathyroid adenoma. Surgery was performed and the histopathological examination of the nodule led to the diagnosis of parathyroid microcarcinoma, sustained by the evidence of vascular invasion, areas of hemorrhage and necrosis and by ancillary studies indicating a Ki67 proliferation index of 55\%; malignant cells in close contact with the surgical resection margin were identified. Postoperative follow-up revealed persisting elevated levels of PTH and calcium and in 2019 a more radical intervention was carried out. The surgical specimen comprised the left thyroid lobe, the remaining left parathyroid gland, the tumoral bed of the previous lesion and local lymph nodes. The histopathology report established the diagnosis of recurrent parathyroid carcinoma with foci of haemorrhage, necrosis, massively infiltrating the left thyroid lobe, the surrounding skeletal muscles and adipose tissue. 15 out of 21 lymph nodes that were examined proved to have carcinomatous cells, some of them even exhibiting capsular effraction. Moreover, the surgical margins of the specimen presented evidence of tumoral invasion, so another intervention was performed. The excision of the remaining right tyroid lobe, of one of the right parathyroid glands and a thorough dissection of the anterior cervical region were later carried out, thus achieving the radicality of the surgical procedure. This last specimen revealed the same diagnosis, along with numerous lymph node metastases in the prethyroid and subthyroid regions (15 out of 21 examined), as well as soft tissue invasion. A few months later during the same year, the patient was admitted in the orthopaedics department for a right femur diaphysis fracture for which he has undergone an intramedullary osteosynthesis with gamma nail. The radiology investigations accomplished on this occasion revealed a mass at the site of the fracture which was biopsied, revealing a reparative process without any malignant elements. Imunohistochemical staining for AE1/AE3 was negative on these frag- 
ments. The patient was then admitted in the oncology department in order to initiate the adjuvant treatment with chemo- and radiotherapy. In 2021, another gamma scan was performed, this time identifying a hyperfixating lesion located in the right paratracheal region, which was then excised and examined. Upon microscopic examination, massive carcinomatous invasion of two lymph nodes and of thymus remnants was identified. Subsequent imunohistochemical investigation of this mass revealed positivity for GATA-3, synaptophysin and chromogranin, as well as negative results for PTH, TTF-1 and PAX8. The value of the Ki-67 index was $30 \%$. Thus, by correlating the history of the patient with the microscopic appearance and with the ancillary studies that were performed, the diagnosis of metastatic parathyroid carcinoma was established. Within one month after surgery, high blood alkaline phosphatase levels were identified in our patient, raising suspicion for distant metastases.

\section{DISCUSSION}

Carcinoma of the parathyroid gland is an outstandingly rare endocrine neoplasm ${ }^{1,2,5}$. However, there has been observed an increase in its incidence in the last decades, partly because of the evolution of criteria required for diagnosis and because of an improved screening, but also because of a real increase in the occurrence of the disease ${ }^{3,5}$.An increased incidence of parathyroid carcinoma has been observed in patients diagnosed with the hyperparathyroidism-jaw tumour syndrome and a strong association with familial isolated hyperparathyroidism has been noted ${ }^{4,5,6}$. Moreover, there are several case reports about malignant epithelial tumours of the parathyroid gland affecting patients diagnosed with multiple endocrine neoplasia type 1 (MEN1) or 2 (MEN 2) 6 . The mean age of patients at the time of the diagnosis is 56 years and the male-to-female ratio is roughly $1: 1^{1,5,6,7}$. Although the data upon etiology of sporadic parathyroid carcinoma is scarce, particularly due to the rarity of this neoplasm, a genetic involvement has been documented for some of the familial forms of the disease, as well as for some small series of cases with sporadic occurrence $5,6,7$.

This malignancy has a highly variable clinical presentation, but most of the signs and symptoms occur as result of elevated levels of PTH and subsequent hypercalcaemia, ranging from mild muscular or digestive problems to life-threatening conditions such as pancre- atitis or cardiac arrhythmias ${ }^{1,7,8}$. Upon admission, many patients show anaemia, signs of chronic kidney disease, some degree of nephrolithiasis and even acute kidney injury $y^{6,7,8}$. Another frequent finding in these patients is bone disease, consisting of lesions such as osteitis fibrosa cystica, resorbtion of the subperiostal bone tissue, osteoporosis, with the latter often leading to fractures ${ }^{3,8}$. Other commonly encountered signs and symptoms are weakness, nausea, vomiting, anorexia, fatigue, polyuria, polydipsia ${ }^{8,9}$. Some other clinical findings, such as a palpable mass in the neck or paralysis of the recurrent laryngeal nerve, are highly suggestive of parathyroid carcinoma, provided they accompany the clinical setting of hyperparathyroidism, which can also be encountered in parathyroid adenomas ${ }^{8,9,10}$. In our patient's case, severe signs and symptoms related to hyperparathyroidism were noted and they especially consisted of renal and osseous lesions. In this particular situation, the good knowledge of the patient's history of recurrent parathyroid neoplasm was crucial in establishing the right diagnosis and adequate treatment.

Even thoughacteristics and genetic features of parathyroid carcinoma are various and this diagnosis is frequently challenging, requiring ancillary studies and a diligent correlation with the clinical course of the disease ${ }^{10,11}$. Moreover, there is a considerable interobserver variability when it comes to differentiating parathyroid adenomas from carcinomas ${ }^{4,11,12}$. As a result, this type of endocrine neoplasm is prone to be misdiagnosed as a parathyroid adenoma $a^{1,2,4,12}$. On the other hand, a small proportion of the lesions initially diagnosed as carcinomas had a truly malignant behaviour on follow-up ${ }^{10,11,12}$. Despite the fact that there are a host of criteria that have been used for differential diagnosis, such as „the triad" composed of macronucleoli, areas of tumour necrosis and more than 5 mitoses/50 HPF, as well as the assessment of capsular or angiolymphatic invasion, the unequivocal histopathological diagnosis of parathyroid carcinoma is often established only when recurrent or metastatic lesions occur ${ }^{10,12}$. Furthermore, immunohistochemical study of primary parathyroid neoplasms may not provide specific data about the malignant nature of the lesion, nor predict the outcome of the disease. In some cases, metastases can be identified at the moment of diagnosis, as their presence is often required for establishing the malignant behaviour of a primary epithelial tumour of the parathyroid gland ${ }^{8,10,13}$. However, there are patients who develop secondary lesions several years after they are diagnosed and treated ${ }^{8}$. This 
particular malignant epithelial proliferation usually spreads by means of local extension or lympho-vascular dissemination, most commonly to cervical or mediastinal lymph nodes, as identified in our patient's case. Metastatic disease should mostly be investigated using gamma scan, followed by surgical excision or biopsy, with histopathological examination of the specimen ${ }^{11,13}$.

An overwhelming majority of these tumours are functional, meaning that the elevated levels of PTH that are produced will be responsible for the clinical picture that accompanies this lesion ${ }^{2,8,14}$. Although PTH is also elevated in parathyroid adenomas, its values are usually only slightly elevated and have a minimal clinical impact ${ }^{14,15}$. Therefore, PTH levels have a vital role in the context of parathyroid tumours, either benign or malignant, starting from the suspicion of a parathyroid lesion and going all the way to the post-therapeutic follow-up. However, there is a small subset of non-functioning tumours, which have been shown to display a more aggressive behaviour. In our patient's case, both the primary tumour and the later recurrent and metastatic lesions were associated with symptomatic hyperparathyroidism, facilitating the identification of the tumour and a proper post-operative monitoring of the disease $\mathrm{s}^{1,8,15}$.

These lesions can have a variable macroscopic appearance, but they usually are large, poorly circumscribed masses with lobular appearance, weighing up to 50 grams. They tend to adhere to the surrounding tissues and are firm and pink-tan on cross section ${ }^{3,4,8,15}$.

These tumors are variably cellular and often exhibit thick fibrous bands, which are responsible for the lobular appearance of the lesion ${ }^{13,14,15}$. The malignant proliferation is mostly composed of chief cells of intermediate size with a predominantly solid or trabecular growth pattern and with round or ovoid hiperchromatic nuclei ${ }^{9,15,16}$. Oncocytes, spindle cells or clear cells tend to be found more rarely ${ }^{12,17}$. Pleomorphism and mitotic figures are usually encountered in a variable degree, but they are of little help in differentiating a malignant lesion from an adenoma ${ }^{13,14,17}$. Atypical mitoses are a strong indicator of malignancy ${ }^{18,19}$. Ki-67 has also been used in this differential diagnosis, but it has a weak diagnostic value in equivocal cases ${ }^{11,19}$. A triad comprised of macronucleoli, areas of tumour necrosis and more than 5 mitoses $/ 50 \mathrm{HPF}$ has been proved to be effective in predicting the aggressive behaviour of these tumours ${ }^{20}$. Some of the positive stains for parathyroid carcinomas are PTH (usually being less expressed than in adenomas), GATA3, CAM5.2, synaptophysin and chromogranin ${ }^{20,21}$.

One of the peculiarities of our patient was that although the primary lesion has proven to be positive for the PTH staining, the mediastinal mass that was discovered upon follow-up imaging turned out to be negative for this staining, which is an exceptional rare finding. As a result, the diagnosis has proven to be a lot more challenging than expected and therefore the history of the patient, as well as the ancillary studies performed were of great value in certifying the final diagnosis. To begin with, the main differential diagnosis which had to be considered was an atypical parathyroid adenoma developed on ectopic parathyroid tissue in the mediastinum. GATA 3 marker is considered very useful in the study of metastatic tumours ${ }^{8,21,22}$. Identifying the expression of this marker in metastatic endocrine neoplasms usually favours their parathyroid origin as part of a panel which must also include Ki 67 proliferation index ${ }^{8,11,22}$. To go on, special stains for pulmonary and thyroid tissue are helpful in excluding these frequent origins of malignant mediastinal proliferations ${ }^{8,19,22}$. In our patient's case, intense nuclear GATA-3 staining in the tumour cells was noted and $\mathrm{Ki} 67$ index was positive in $30 \%$ of the tumour cells, highly suggesting the parathyroid origin of the lesion. Later, chromogranin and synaptophysin staining was performed and intense expression was noted. TTF- 1 and PAX- 8 negativity ruled out the pulmonary or thyroid origin of the tumour. Consequently, the diagnosis of metastatic parathyroid carcinoma was established.

Considering all these aspects, we emphasize the importance of an adequate collaboration between departments, both clinical and paraclinical, as it provides the best possible care for patients. We also underline the importance of a thorough medical history and a rigorous follow-up, especially for lesions with an uncertain or unpredictable malignant potential. The mainstay of treatment is aggressive local resection, for primary, relapsing or metastatic tumours. Medical management of inoperable disease is aimed at reducing the life-threatening hypercalcaemia using calcimimetics such as Cinacalcet and has proved to be relatively effective. Chemotherapy has also proved to effective, but with limited evidence.

\section{CONCLUSION}

The diagnosis and treatment of malignant parathyroid lesions can be challenging, especially considering their 
unusual histopathological aspect and their various clinical presentation, as well as their rarity/uncommonness. Although such endocrine neoplasms represent exceptional clinical findings, the positive and differential diagnosis should be correctly established, as surgical treatment and oncological treatment can prolong the patient's survival and alleviate the symptoms.
Compliance with ethics requirements: The authors declare no conflict of interest regarding this article. The authors declare that all the procedures and experiments of this study respect the ethical standards in the Helsinki Declaration of 1975, as revised in 2008(5) and the national law. Informed consent was obtained from the patient described in the clinical case and his parents.
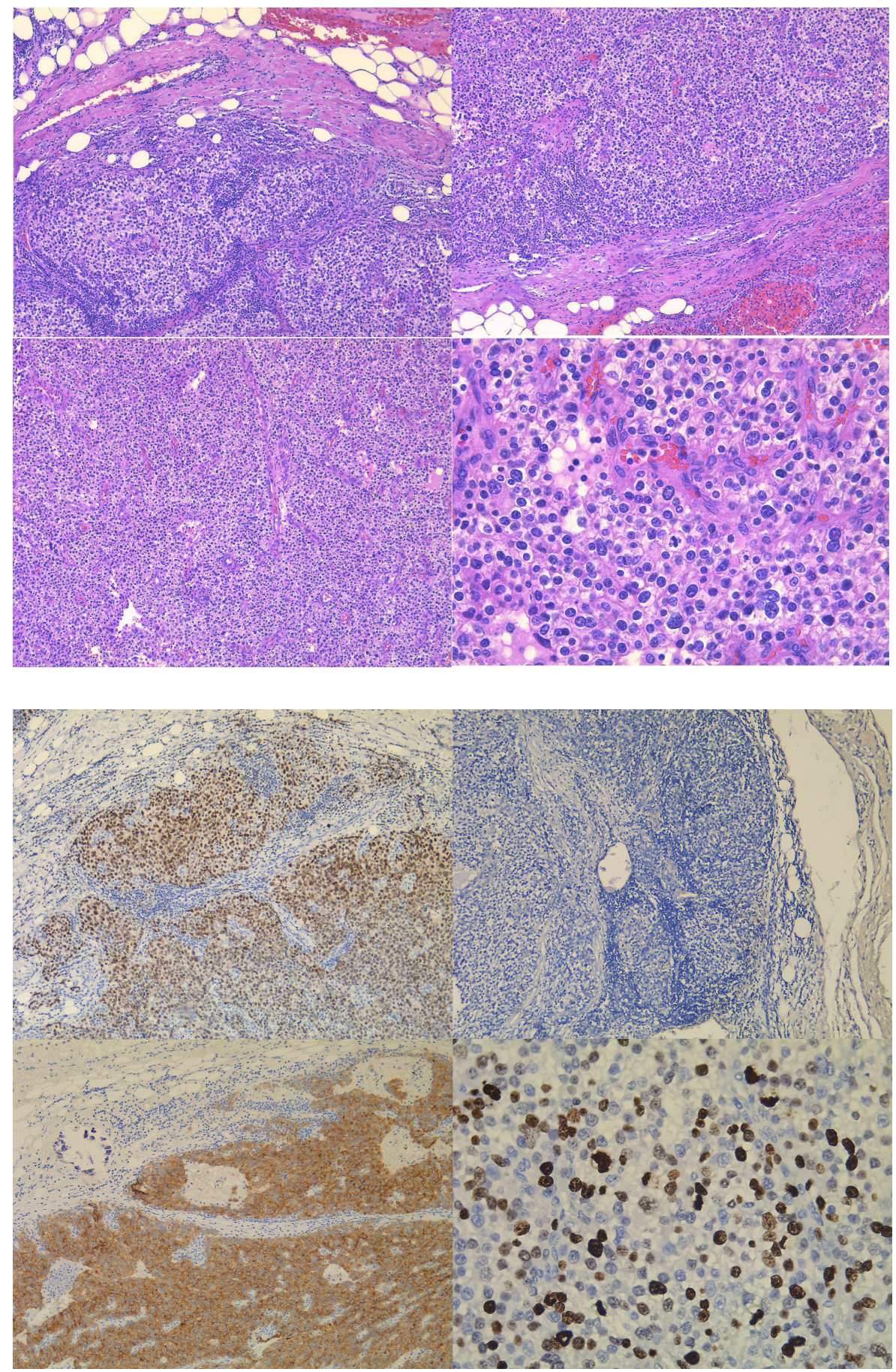

Figure 1. Metastatic parathyroid carcinoma involving mediastinal lymph nodes. Up left, up right and down left panels: Malignant epithelial proliferation composed of atypical intermediate-sized cells, predominantly exhibiting a solid growth pattern, with no evidence of vascular or perineural invasion, HE $10 \mathrm{X}$, HE, 20 X; Downright panel: Moderately pleomorphic nuclei and atypical mitotic figures $\mathrm{HE}, 40 \mathrm{X}$.

Figure 2. Up left panel: Intense nuclear positivity of GATA 3 marker within tumour cells; Up right panel: Negative PTH immunostaining within tumour cells; Down left panel: Positive cytoplasmic synaptophysin stain; Downright panel: Expression of Ki 67 proliferation index in $30 \%$ of the tumour cells. 


\section{References}

1. Goswamy J, Lei M, Simo R. Parathyroid carcinoma. Curr Opin Otolaryngol Head Neck Surg. 2016 Apr;24(2):155-62. doi: 10.1097/ M00.0000000000000234. PMID: 26771263.

2. Mohebati A, Shaha A, Shah J. Parathyroid carcinoma: challenges in diagnosis and treatment. Hematol Oncol Clin North Am. 2012 Dec;26(6):1221-38. doi: 10.1016/j.hoc.2012.08.009. Epub 2012 Oct 5. PMID: 23116578

3. Kassahun WT, Jonas S. Focus on parathyroid carcinoma. Int J Surg. 2011;9(1):13-9. doi: 10.1016/j.ijsu.2010.09.003. Epub 2010 Sep 30. PMID: 20887820.

4. Wiseman SM, Rigual NR, Hicks WL Jr, Popat SR, Lore JM Jr, Douglas WG, Jacobson MJ, Tan D, Loree TR. Parathyroid carcinoma: a multicenter review of clinicopathologic features and treatment outcomes. Ear Nose Throat J. 2004 Jul;83(7):491-4. PMID: 15372923.

5. Sali AP, Motghare P, Bal M, Mittal N, Rane S, Kane S, Patil A. Parathyroid Carcinoma: A Single-Institution Experience with an Emphasis on Histopathological Features. Head Neck Pathol. 2021 Jun;15(2):544-554. doi: 10.1007/s12105-020-01244-x. Epub 2020 Nov 5. PMID: 33151464; PMCID: PMC8134611.

6. Cetani F, Pardi E, Marcocci C. Parathyroid carcinoma: a clinical and genetic perspective. Minerva Endocrinol. 2018 Jun;43(2):144155. doi: 10.23736/S0391-1977.17.02737-7. Epub 2017 Sep 25. PMID: 28949121

7. Twigt BA, van Dalen T, Vroonhoven TJ, Consten EC. Recurrent hyperparathyroidism caused by benign neoplastic seeding: two cases of parathyromatosis and a review of the literature. Acta Chir Belg. 2013 May-Jun;113(3):228-32. doi: 10.1080/00015458.2013.11680918. PMID: 24941723.

8. Li J, Chen W, Liu A. [Clinicopathologic features of parathyroid carcinoma: a study of 11 cases with review of literature]. Zhonghua Bing Li Xue Za Zhi. 2014 May;43(5):296-300. Chinese. PMID: 25030860.

9. Chien $\mathrm{D}$, Jacene $\mathrm{H}$. Imaging of parathyroid glands. Otolaryngol Clin North Am. 2010 Apr;43(2):399-415, x. doi: 10.1016/j. otc. 2010.01.008. PMID: 20510723.

10. Cetani F, Pardi E, Marcocci C. Parathyroid Carcinoma. Front Horm Res. 2019; 51:63-76. doi: 10.1159/000491039. Epub 2018 Nov 19. PMID: 30641523.

11. Cetani F, Pardi E, Marcocci C. Update on parathyroid carcinoma. J Endocrinol Invest. 2016 Jun;39(6):595-606. doi: 10.1007/s40618016-0447-3. Epub 2016 Mar 21. PMID: 27001435.

12. Schulte JJ, Pease G, Taxy JB, Hall C, Cipriani NA. Distinguishing Parathyromatosis, Atypical Parathyroid Adenomas, and Parathyroid Carcinomas Utilizing Histologic and Clinical Features. Head Neck Pathol. 2021 Sep;15(3):727-736. doi: 10.1007/s12105020-01281-6. Epub 2021 Jan 4. PMID: 33394375; PMCID: PMC8384997.
13. Troilo VL, D'Eredità G, Fischetti F, Berardi T. Parathyroid cancer as rare cause of primary hyperparathyroidism. Case report and review of the literature. G Chir. 2009 Oct;30(10):432-6. PMID: 19954585.

14. Mendoza V, Hernández AF, Márquez ML, Delgadillo MA, Peña J, Mercado M. Primary hyperparathyroidism due to parathyroid carcinoma. Arch Med Res. 1997 Summer;28(2):303-6. PMID: 9204627.

15. Chang YJ, Mittal V, Remine S, Manyam H, Sabir M, Richardson T, Young S. Correlation between clinical and histological findings in parathyroid tumors suspicious for carcinoma. Am Surg. 2006 May;72(5):419-26. PMID: 16719197.

16. Wells SA Jr, Debenedetti MK, Doherty GM. Recurrent or persistent hyperparathyroidism. J Bone Miner Res. 2002 Nov;17 Suppl 2: N158-62. PMID: 12412795.

17. Abruzzo A, Gioviale MC, Damiano G, Palumbo VD, Buscemi S, Lo Monte G, Gulotta L, Buscemi G, Lo Monte Al. Reoperation for persistent or recurrent secondary hyperparathyroidism. Acta Biomed. 2017 Oct 23;88(3):325-328. doi: 10.23750/abm. v88i3.4722. PMID: 29083339; PMCID: PMC6142843.

18. Boddi W, Nozzoli C, Francois C, Amorosi A, Grifoni S, Morettini A Olivotto J, Berni G. Iperparatiroidismo da carcinoma delle paratiroidi a sede mediastinica [Hyperparathyroidism due to parathyroid carcinoma located in the mediastinum]. Ann Ital Med Int. 1994 Jan-Mar;9(1):32-4. Italian. PMID: 8003390.

19. Lappas D, Noussios G, Anagnostis P, Adamidou F, Chatzigeorgiou A, Skandalakis P. Location, number and morphology of parathyroid glands: results from a large anatomical series. Anat Sci Int 2012 Sep;87(3):160-4. doi: 10.1007/s12565-012-0142-1. Epub 2012 Jun 12. PMID: 22689148.

20. Chen J, Wan Y, Chen S. Rare concurrence of ectopic intrathyroidal parathyroid gland and papillary thyroid carcinoma within a thyroid lobe: A care-compliant case report. Medicine (Baltimore). 2019 Aug;98(34): e16893. doi: 10.1097/MD.0000000000016893. PMID: 31441867; PMCID: PMC6716706.

21. Balakrishnan M, George SA, Rajab SH, Francis IM, Kapila K. Cytological challenges in the diagnosis of intrathyroidal parathyroid carcinoma: A case report and review of literature. Diagn Cytopathol. 2018 Jan;46(1):47-52. doi: 10.1002/dc.23847. Epub 2017 Oct 27. PMID: 29076656.

22. Lee KM, Kim EJ, Choi WS, Park WS, Kim SW. Intrathyroidal parathyroid carcinoma mimicking a thyroid nodule in a MEN type 1 patient. J Clin Ultrasound. 2014 May;42(4):212-4. doi: 10.1002/ jcu.22090. Epub 2013 Sep 4. PMID: 24037737. 